\title{
Trades in complex Hadamard matrices
}

\author{
PAdRAig Ó CATHÁIN * \\ IAN M. WANLESS ${ }^{\dagger}$ \\ School of Mathematical Sciences, \\ Monash University, VIC 3800, Australia.
}

July 26, 2018

\begin{abstract}
A trade in a complex Hadamard matrix is a set of entries which can be changed to obtain a different complex Hadamard matrix. We show that in a real Hadamard matrix of order $n$ all trades contain at least $n$ entries. We call a trade rectangular if it consists of a submatrix that can be multiplied by some scalar $c \neq 1$ to obtain another complex Hadamard matrix. We give a characterisation of rectangular trades in complex Hadamard matrices of order $n$ and show that they all contain at least $n$ entries. We conjecture that all trades in complex Hadamard matrices contain at least $n$ entries.
\end{abstract}

2010 Mathematics Subject classification: 05B20, 15B34

Keywords: Hadamard matrix, trade, rank

\footnotetext{
${ }^{0}$ This work was inspired by the discussion after Will Orrick's talk at the ADTHM'14 workshop, and much of the work was undertaken at the workshop. The authors are grateful to the workshop organisers and to BIRS. Research supported by ARC grants FT110100065 and DP120103067. This is the final form of this work. No other version has been or will be submitted elsewhere.

*E-mail: p.ocathain@gmail.com

†E-mail: ian.wanless@monash.edu
} 


\section{Introduction}

A complex Hadamard matrix of order $n$ is an $n \times n$ complex matrix with unimodular entries which satisfies the matrix equation

$$
H H^{\dagger}=n I_{n},
$$

where $H^{\dagger}$ is the conjugate transpose of $H$ and $I_{n}$ is the $n \times n$ identity matrix. If the entries are real (hence \pm 1 ) the matrix is Hadamard. The notion of a trade is well known in the study of $t$-designs and Latin squares [1]. For a complex Hadamard matrix we define a trade to be a set of entries which can be altered to obtain a different complex Hadamard matrix of the same order. In other words, a set $T$ of entries in a complex Hadamard matrix $H$ is a trade if there exists another complex Hadamard matrix $H^{\prime}$ such that $H$ and $H^{\prime}$ disagree on every entry in $T$ but agree otherwise. If $H$ is a real Hadamard matrix, we insist that $H^{\prime}$ is also real.

Example 1. The 8 shaded entries in the Paley Hadamard matrix below form a trade.

$$
\left(\begin{array}{cccccccc}
+ & + & + & + & + & + & + & + \\
+ & - & - & - & + & - & + & + \\
+ & + & - & - & - & + & - & + \\
+ & + & + & - & - & - & + & - \\
+ & - & + & + & - & - & - & + \\
+ & + & - & + & + & - & - & - \\
+ & - & + & - & + & + & - & - \\
+ & - & - & + & - & + & + & -
\end{array}\right)
$$

If each of the shaded entries is replaced by its negative, the result is another Hadamard matrix.

We use the word switch to describe the process of replacing a trade by a new set of entries (which must themselves form a trade). In keeping with the precedent from design theory, our trades are simply a set of entries that can be switched. Information about what they can be switched to does not form part of the trade (although it may be helpful in order to see that something is a trade). For real Hadamard matrices there can only be one way to switch a given trade, since only two symbols are allowed in the matrices and switching must change every entry in a trade. However, for complex Hadamard matrices there can be more than one way to switch a given trade, as our next example shows.

Example 2. Let $u$ be a nontrivial third root of unity. The following matrix is a $7 \times 7$ complex Hadamard matrix. The shaded entries again form a trade; they can be multiplied by an arbitrary complex number $c$ of modulus 1 to obtain another complex Hadamard matrix. This matrix is due originally to Petrescu [6], and is available in the online database [2].

$$
\left(\begin{array}{cccrrrr}
1 & 1 & 1 & 1 & 1 & 1 & 1 \\
1 & -u & u & -u^{2} & -1 & -1 & -u \\
1 & u & -u & -1 & -u^{2} & -1 & -u \\
1 & -u^{2} & -1 & u & -u & -u & -1 \\
1 & -1 & -u^{2} & -u & u & -u & -1 \\
1 & -1 & -1 & -u & -u & u & -u^{2} \\
1 & -u & -u & -1 & -1 & -u^{2} & u
\end{array}\right)
$$


The size of a trade is the number of entries in it. We say that a trade is rectangular if the entries in the trade form a submatrix that can be switched by multiplying all entries in the trade by some complex number $c \neq 1$ of unit modulus. It will follow from Lemma 3 that the value of $c$ is immaterial; if one value works then they will all work. In a complex Hadamard matrix each row and column is a rectangular trade. Thus there are always $1 \times n$ and $n \times 1$ rectangular trades. Similarly, we may exchange any pair of rows to obtain another complex Hadamard matrix. In the real case, the rows that we exchange necessarily differ in exactly half the columns, so this reveals a $2 \times \frac{n}{2}$ rectangular trade (and similarly there are always $\frac{n}{2} \times 2$ rectangular trades in real Hadamard matrices). Less trivial trades were used by Orrick [5] to generate many inequivalent Hadamard matrices of orders 32 and 36. The smaller of Orrick's two types of trades was a $4 \times \frac{n}{4}$ rectangular trade that he called a "closed quadruple". Closed quadruples are often but not always present in Hadamard matrices. The trades just discussed all have size equal to the order $n$ of the host matrix. The trade in Example 1 is a non-rectangular example with the same property.

Trades in real Hadamard matrices and related codes and designs have been studied occasionally in the literature, either to produce invariants to aid with classification or to produce many inequivalent Hadamard matrices. See [5] and the references cited there. In the complex case, trades are related to parameterising complex Hadamard matrices, some computational and theoretical results are surveyed in [7].

Throughout this note we will assume that $H=\left[h_{i j}\right]$ is a complex Hadamard matrix of order $n$. We will use $r_{i}$ and $c_{j}$ to denote the $i$-th row and $j$-th column of $H$ respectively. If $B$ is a set of columns then $r_{i, B}$ denotes the row vector which is equal to $r_{i}$ on the coordinates $B$ and zero elsewhere. We use $\bar{B}$ for the complement of the set $B$.

\section{$2 \quad$ Hadamard Trades}

We start with a basic property of trades. We use $\langle\cdot, \cdot\rangle$ for the standard Hermitian inner product under which rows of a complex Hadamard matrix are orthogonal.

Lemma 3. Let $T$ be a subset of the entries of a complex Hadamard matrix $H$. Let $c \neq 1$ be a complex number of unit modulus.

1. Suppose that $T$ can be switched by multiplying its entries by $c$. Let $B$ be the set of columns in which row $r_{i}$ of $H$ contains elements of $T$. If $r_{j}$ is a row of $H$ that contains no elements of $T$ then $r_{i, B}$ is orthogonal to $r_{j, B}$.

2. Suppose that $T$ forms a rectangular submatrix of $H$ with rows $A$ and columns $B$. Then $T$ can be switched by multiplying its entries by $c$ if and only if $r_{i, B}$ is orthogonal to $r_{j, B}$ for every $r_{i} \in A$ and $r_{j} \notin A$.

Proof. First, since the rows of $H$ are orthogonal, we have that

$$
0=\left\langle r_{i}, r_{j}\right\rangle=\left\langle r_{i, B}, r_{j, B}\right\rangle+\left\langle r_{i, \bar{B}}, r_{j, \bar{B}}\right\rangle .
$$

Now, multiplying the entries in $T$ by $c$, we see that

$$
0=\left\langle c r_{i, B}, r_{j, B}\right\rangle+\left\langle r_{i, \bar{B}}, r_{j, \bar{B}}\right\rangle=c\left\langle r_{i, B}, r_{j, B}\right\rangle+\left\langle r_{i, \bar{B}}, r_{j, \bar{B}}\right\rangle .
$$


Subtracting, we find that $(c-1)\left\langle r_{i, B}, r_{j, B}\right\rangle=0$. Given that $c \neq 1$ the first claim of the Lemma follows.

We have just shown the necessity of the condition in the second claim. To check sufficiency we note that the above argument is reversible and shows that $r_{i} \in A$ and $r_{j} \notin A$ will be orthogonal after multiplication of the entries of $T$ by $c$. So we just have to verify that any two rows $r_{i}, r_{k}$ in $A$ will be orthogonal. This follows from

$$
\left\langle c r_{i, B}, c r_{k, B}\right\rangle+\left\langle r_{i, \bar{B}}, r_{k, \bar{B}}\right\rangle=|c|\left\langle r_{i, B}, r_{k, B}\right\rangle+\left\langle r_{i, \bar{B}}, r_{k, \bar{B}}\right\rangle=\left\langle r_{i, B}, r_{k, B}\right\rangle+\left\langle r_{i, \bar{B}}, r_{k, \bar{B}}\right\rangle=0 .
$$

Note that the value of $c$ plays no role in Lemma 3. Also, Part 1 of the lemma implies that in a real Hadamard matrix any trade which does not intersect every row must use an even number of entries from each row. The same is not true for trades in complex Hadamard matrices (see [2] for examples).

It is of interest to consider the size of a smallest possible trade. For (real) Hadamard matrices of order $n$ we show that arbitrary trades have size at least $n$. Equality is achievable in a variety of ways, as discussed above. However, we find a restriction that must be obeyed by any trade achieving equality. Then we show that in the general case rectangular trades have size at least $n$. The question for arbitrary trades in complex Hadamard matrices remains open.

Theorem 4. Let $H$ be a (real) Hadamard matrix of order $n$. Any trade in $H$ has size at least $n$. If $T$ is any trade of size $n$ in $H$ then there are divisors $d$ and $e$ of $n$ such that $T$ contains either 0 or $d$ entries in each row of $H$ and either 0 or e entries in each column of $H$. Moreover, $d$ is even or $d=1$. Likewise, $e$ is even or $e=1$.

Proof. Suppose that $H$ differs from a Hadamard matrix $H^{\prime}$ in a trade $T$ of at most $n$ entries. Without loss of generality, we assume that $H$ is normalised, that the first row of $H$ contains $d$ differences between $H$ and $H^{\prime}$, and that these differences occur in the first $d$ columns. We also assume that all differences between $H$ and $H^{\prime}$ occur in the first $r$ rows, with each of those rows having at least $d$ differences in them. The case $r=n$ is trivial, so we assume that $r<n$ in the remainder of the proof. By assumption there are at least $r d$ entries in $T$, so $r d \leqslant n$. Now consider the submatrix $S$ of $H$ formed by the first $d$ columns and the last $n-r$ rows. By Lemma 3, we know that each row of $S$ is orthogonal to the all ones vector. It follows that $d$ is even and $S$ contains $(n-r) d / 2$ negative entries. The first column of $S$ consists entirely of ones so, by the pigeon-hole principle, some other column of $S$ must contain at least

$$
\frac{(n-r) d}{2(d-1)} \leqslant \frac{n d-n}{2(d-1)}=\frac{n}{2}
$$

negative entries. This column of $H$ is orthogonal to the first column, so we must have equality in (1). It follows that $n=r d$ and each of the first $r$ rows contain exactly $d$ entries in $T$. Columns have similar properties, by symmetry.

Corollary 5. In a (real) Hadamard matrix of order $n$ the symmetric difference of any two trades must have size at least $n$. 
Proof. Suppose that $H, H_{1}, H_{2}$ are distinct (real) Hadamard matrices of order $n$. Let $T_{1}$ and $T_{2}$ be the set of entries of $H$ which disagree with the corresponding entries of $H_{1}$ and $H_{2}$ respectively. The symmetric difference of $T_{1}$ and $T_{2}$ has cardinality equal to the number of entries of $H_{1}$ that are different to the corresponding entry of $H_{2}$. This cardinality is at least $n$, by Theorem 4 .

Example 1 is the symmetric difference of two rectangular trades, one $2 \times 4$ and the other $4 \times 2$. It shows that equality can be achieved in the Corollary. The example also demonstrates that trades of minimal size need not be rectangular. In the notation of Theorem 4 it has $d=e=2$ and $n=8$. Another example is obtained as follows. Let $H$ be any Hadamard matrix and $H^{\prime}$ the matrix obtained by swapping two rows of $H$, then negating one of the rows that was swapped. Let $T$ be the trade consisting of the entries of $H$ which differ from the corresponding entry in $H^{\prime}$. It is easy to show that $T$ has $d=n / 2, e=1$ in the notation of Theorem 4 .

It is also possible to have $d=e=1$. If this is the case then by permuting and/or negating rows we obtain a Hadamard matrix $H$ for which $H-2 I$ is also Hadamard, where $I$ is the identity matrix. However this means that

$$
H H^{\top}=(H-2 I)(H-2 I)^{\top}=H H^{\top}-2 H-2 H^{\top}+4 I .
$$

Hence $H+H^{\top}=2 I$, so $H$ is a skew-Hadamard matrix. Conversely, the main diagonal of any skew-Hadamard matrix is a trade with $d=e=1$.

Now we consider complex Hadamard matrices. The following lemma is the key step in our proof. The corresponding result for real Hadamard matrices has been obtained by Alon (cf. [10, Lemma 14.6). Alon's proof can be trivially adapted to deal with complex Hadamard matrices. We include our own independent proof here since we want to extract a characterisation of cases where the bound is tight.

Lemma 6. Let $H$ be a complex Hadamard matrix of order $n$, and $B$ a set of $b$ columns of $H$. If $\alpha$ is a non-zero linear combination of the elements of $B$ then $\alpha$ has at least $\left\lceil\frac{n}{b}\right\rceil$ non-zero entries.

Proof. Without loss of generality, we can write $H$ in the form

$$
H=\left(\begin{array}{ll}
T & U \\
V & W
\end{array}\right)
$$

where $T$ contains the columns in $B$ and the rows in which $\alpha$ is non-zero. We will identify a linear dependence among the rows of $U$, then use this and an expression for the inner product of $r_{1}$ and $r_{2}$ to derive the required result. We assume that there are $t$ non-zero entries $\alpha_{i}$ in $\alpha$ and that if $t \geqslant 2$ then they obey $\left|\alpha_{2}\right| \geqslant\left|\alpha_{1}\right| \geqslant\left|\alpha_{i}\right|$ for $3 \leqslant i \leqslant t$. We need to show that $t \geqslant\left\lceil\frac{n}{b}\right\rceil$.

For any column $c_{j}$ not in $B$, we have that $\left\langle c_{j}, \alpha\right\rangle=0$ since the columns of $H$ are orthogonal. Thus every column of $U$ is orthogonal to $\alpha$, and so there exists a linear dependence among the rows of $U$, explicitly: $h_{1 j}=\sum_{i=2}^{t}-\alpha_{i} \alpha_{1}^{-1} h_{i j}$, for any $j \notin B$. In particular, this shows that indeed $t \geqslant 2$. 
Since $H$ is Hadamard, we know that all of the $h_{i j}$ have absolute value 1 , and that rows of $H$ are necessarily orthogonal:

$$
\begin{aligned}
\left\langle r_{1}, r_{2}\right\rangle & =\left\langle r_{1, B}, r_{2, B}\right\rangle+\left\langle r_{1, \bar{B}}, r_{2, \bar{B}}\right\rangle \\
& =\left\langle r_{1, B}, r_{2, B}\right\rangle+\left\langle\sum_{i=2}^{t}-\alpha_{i} \alpha_{1}^{-1} r_{i, \bar{B}}, r_{2, \bar{B}}\right\rangle \\
& =\left\langle r_{1, B}, r_{2, B}\right\rangle+\sum_{i=2}^{t}-\alpha_{i} \alpha_{1}^{-1}\left\langle r_{i, \bar{B}}, r_{2, \bar{B}}\right\rangle .
\end{aligned}
$$

Since $\left\langle r_{1}, r_{2}\right\rangle=0$ and $\left\langle r_{i, \bar{B}}, r_{2, \bar{B}}\right\rangle=-\left\langle r_{i, B}, r_{2, B}\right\rangle$, this means that

$$
\alpha_{2} \alpha_{1}^{-1}\left\langle r_{2, \bar{B}}, r_{2, \bar{B}}\right\rangle=\left\langle r_{1, B}, r_{2, B}\right\rangle+\sum_{i=3}^{t} \alpha_{i} \alpha_{1}^{-1}\left\langle r_{i, B}, r_{2, B}\right\rangle .
$$

Now, each inner product $\left\langle r_{i, B}, r_{2, B}\right\rangle$ is a sum of $b$ complex numbers of modulus one, and $\left|\alpha_{i} \alpha_{1}^{-1}\right| \leqslant 1$ for $i \geqslant 3$. So the absolute value of the right hand side of (2) is at most $(t-1) b$. In contrast, the absolute value of the left hand side of (2) is $\left|\alpha_{2} \alpha_{1}^{-1}\right|(n-b) \geqslant n-b$. It follows that $n-b \leqslant(t-1) b$, and hence $t \geqslant\left\lceil\frac{n}{b}\right\rceil$.

Let $H$ be a Fourier Hadamard matrix of order $n$, and suppose that $t \mid n$. Then there exist $t$ rows of $H$ containing only $t^{\text {th }}$ roots of unity. Their sum vanishes on all but $\frac{n}{t}$ coordinates, so Lemma 6 is best possible. On the other hand, if $H$ is Fourier of prime order $p$, the only vanishing sum of $p^{\text {th }}$ roots is the complete one. So in this case, a linear combination of at most $t$ rows will contain at most $t$ zero entries.

Theorem 7. If $H$ is a complex Hadamard matrix of order $n$ containing an $a \times b$ rectangular trade $T$ then $a b \geqslant n$. If $a b=n$ then $T$ is a rank one submatrix of $H$.

Proof. Without loss of generality, $T$ lies in the first $a$ rows of $H$. Let $B$ be the set of the columns that contain the entries of $T$. By hypothesis, $\gamma_{1}=\sum_{1 \leqslant i \leqslant a} r_{i}$ and $\gamma_{c}=\sum_{1 \leqslant i \leqslant a}\left(c r_{i, B}+r_{i, \bar{B}}\right)$ are both orthogonal to the space $U$ spanned by the last $n-a$ rows of $H$. Now consider $\gamma_{1}-\gamma_{c}$, which is zero in any column outside $B$, but which is not zero since the rows of $H$ are linearly independent. Observe that the orthogonal complement of $U$ is $a$-dimensional, and that the initial $a$ rows of $H$ span this space: thus $\gamma_{1}-\gamma_{c}$ is in the span of these rows, Lemma 6 applies, and $a b \geqslant n$.

If $a b=n$ then, equality holds in calculations at the end of the proof of Lemma 6. In particular, $\left|\left\langle r_{i, B}, r_{2, B}\right\rangle\right|=b$ for each $i$, which implies that $r_{i, B}$ is collinear to $r_{2, B}$. Hence $T$ is a rank one submatrix of $H$.

We now give a complete characterisation of the minimal rectangular trades in any complex Hadamard matrix.

Theorem 8. Let $H$ be a complex Hadamard matrix of order $n$ and $T$ an $a \times b$ submatrix of $H$ with $a b=n$. Then $T$ is a rectangular trade if and only if $T$ is rank 1 . 
Proof. Theorem 7 shows that any rectangular trade of size $n$ is necessarily rank one. So we need only prove the converse. Without loss of generality, we assume that $T$ is contained in the first $a$ rows and first $b$ columns of $H$ and that $H$ is normalised. Note that this implies that $T$ is an all ones submatrix.

Consider $\gamma=\left(\gamma_{1}, \ldots, \gamma_{n}\right)=\sum_{i=1}^{b} c_{i}$, the sum of the first $b$ columns of $H$. It is clear that $\gamma_{j}=b$ for $j \in\{1, \ldots, a\}$. If we show that $\gamma_{j}=0$ for $a<j \leqslant n$ then Lemma 3 will show that $T$ is a trade. We calculate the $\ell_{2}$ norm of $\gamma$ in two ways: first, via an expansion into orthogonal vectors:

$$
\|\gamma\|_{2}^{2}=\left\langle\sum_{i=1}^{b} c_{i}, \sum_{i=1}^{b} c_{i}\right\rangle=\sum_{i=1}^{b}\left\langle c_{i}, c_{i}\right\rangle=b n .
$$

On the other hand, $\|\gamma\|_{2}^{2}=\sum_{i=1}^{n}\left|\gamma_{i}\right|^{2}$. We have that $\gamma_{i}=b$ for $1 \leqslant i \leqslant a$. But $a b^{2}=n b$, so $\left|\gamma_{i}\right|=0$ for all $i>a$. Applying Lemma [3, we are done.

Corollary 9. If $T$ is an $a \times b$ rank one submatrix of $H$, then $T$ is a trade if and only if $a b=n$.

Proof. We have that $a b \geqslant n$ by Theorem 7 . In the other direction, Lindsay's Lemma states that the size of a rank one submatrix of a Hadamard matrix of order $n$ is bounded above by $n$ (see Lemma 14.5 of [10]).

Ryser's embedding problem is to establish the minimal order, $R(a, b)$, of a Hadamard matrix containing an $a \times b$ submatrix consisting entirely of ones. Any rank one submatrix can be transformed into a submatrix consisting entirely of ones by a sequence of Hadamard equivalence operations. Hence there is a Hadamard matrix of order $a b$ containing an $a \times b$ rectangular trade if and only if $R(a, b)=a b$.

Newman [8] showed that $R(a, b)=a b$ whenever both $a, b$ are orders for which Hadamard matrices exist. Michael [9] showed that $R(a, b) \geqslant(a+1) b$ for odd $a>1$. Thus there are no $a \times b$ rectangular trades in this case, a conclusion that could also be reached from Theorem 4 . Michael also showed that if $2 a$ and $b / 2$ are orders of Hadamard matrices then there exists an $a \times b$ rectangular trade in a Hadamard matrix of order $a b$. For example, there is a Hadamard matrix of order 48 containing a $6 \times 8$ rectangular trade.

\section{Open questions}

A Bush type Hadamard matrix of order $m^{2}$ contains an $m \times m$ rank one submatrix. Hence there is a Hadamard matrix of order 36 containing a $6 \times 6$ rectangular trade. Thus all cases of our first question smaller than $a=6, b=10$ are resolved.

Question 1: Are there even integers $a, b$ for which there does not exist a Hadamard matrix of order $a b$ containing an $a \times b$ rectangular trade?

On the basis of Theorem 4 and Theorem 7 we are inclined to think that the answer to the following question is negative:

Question 2: Can there exist trades of size less than $n$ in an $n \times n$ complex Hadamard matrix? 
It would also be nice to know how "universal" the rectangular trades we have studied are. Example 1 showed that combinations of rectangular trades can create more complicated trades. By iterating such steps can we build all trades? In other words:

Question 3: Is every trade in a (real) Hadamard matrix a $\mathbb{Z}_{2}$-linear combination of rectangular trades? If so, how does this generalise to the complex case?

This work was motivated in part by problems in the construction of compressed sensing matrices [3]. Optimal complex Hadamard matrices for this application have the property that linear combinations of $t$ rows vanish in at most $t$ components.

Question 4: Other than Fourier matrices, are their families of Hadamard matrices with the property that no linear combination of $t$ rows contains more than $t$ zeros? Or, if such matrices are rare, describe families in which no linear combination of $t$ rows contain more than $f(t)$ zeros for some slowly growing function $f$.

We are indebted to Prof. Robert Craigen for our final question and the accompanying example.

Question 5: To what extent do the results in this paper generalise to weighing matrices (and complex weighing matrices and their generalisations)? In particular, is the weight of a weighing matrix a lower bound on the size of all trades in that matrix? Note that any weighing matrix has a trade of size equal to its weight, simply by negating a row. Slightly less trivially, trades with size equal to the weight can be obtained by weaving (see [4]) weighing matrices. For example, take any $2 \times 2$ block of rank one in the following $\mathrm{W}(6,4)$. The shaded entries show one such block.

$$
\left(\begin{array}{cccccc}
0 & 0 & + & + & + & + \\
0 & 0 & + & + & - & - \\
+ & + & 0 & 0 & + & - \\
+ & + & 0 & 0 & - & + \\
+ & - & + & - & 0 & 0 \\
- & + & - & + & 0 & 0
\end{array}\right)
$$

\section{References}

[1] E. J. Billington, Combinatorial trades: a survey of recent results, W.D. Wallis (ed.), Designs 2002: Further computational and constructive design theory, Kluwer, 2003, pp. 4767.

[2] W. Bruzda, W. Tadej and K. Życzkowski, Catalogue of complex Hadamard matrices, http://chaos.if.uj.edu.pl/ karol/hadamard/, retrieved 10/09/2014.

[3] D. Bryant and P. Ó Catháin, An asymptotic existence result on compressed sensing matrices, submitted, March 2014.

[4] R. Craigen, The craft of weaving matrices, Congr. Numer. 92 (1993), 9-28.

[5] W. P. Orrick, Switching operations for Hadamard matrices, SIAM J. Discrete Math. 22 (2008), 31-50. 
[6] M. Petrescu, Existence of Continuous Families of Complex Hadamard Matrices of Certain Prime Dimensions and Related Results, PhD thesis, University of California, Los Angeles, (1997).

[7] W. Tadej and K. Życzkowski A Concise Guide to Complex Hadamard Matrices, Open Syst. Inf. Dyn., 13 (2006), 133-177.

[8] M. Newman On a problem of H. J. Ryser, Linear Multilinear Algebra, 12 (1982), 291-293.

[9] T. S. Michael, Ryser's embedding problem for Hadamard matrices, J. Combin. Des., 14, (2006), 41-51.

[10] S. Jukna, Extremal combinatorics (2nd ed.), Texts in Theoretical Computer Science, Springer, 2011. 